\title{
Clinical Features and Hearing Outcomes of Sudden Sensorineural Hearing Loss in Diabetic Patients
}

\author{
Yeo Rim Ju, Hyoung-sik Park, Min Young Lee, Jae Yun Jung, and Ji Eun Choi \\ Department of Otorhinolaryngology-Head and Neck Surgery, Dankook University Hospital, Cheonan, Korea
}

Received May 30, 2020

Revised July 16, 2020

Accepted August 27, 2020

\section{Address for correspondence Ji Eun Choi, MD \\ Departement of Otorhinolaryngology- \\ Head and Neck Surgery, \\ Dankook University Hospital, \\ 201 Manghyang-ro, Dongnam-gu, \\ Cheonan 31116, Korea \\ Tel +82-41-550-3284 \\ Fax +82-41-556-1090 \\ E-mail garimung@gmail.com}

Background and Objectives: This study aimed to evaluate the clinical features and the clinical factors associated with prognosis of sudden sensorineural hearing loss (SSNHL) in diabetic patients. Subjects and Methods: Forty-nine diabetic with unilateral SSNHL were retrospectively included. All patients received systemic high dose steroid therapy within one month after onset and had more than one month of follow-up audiogram. The basic characteristics of the patients, initial and follow-up audiograms, laboratory data, and methods of steroid treatment were collected. Results: Compared to reference values in healthy subjects, $79 \%, 55 \%$, and $45 \%$ of the patients had higher values of mean neutrophil-lymphocyte ratio $(\mathrm{NLR})$, platelet-lymphocyte ratio (PLR), and lymphocyte-monocyte ratio (LMR), respectively. Older patients had significantly less degree of hearing loss, but they also had significantly worse hearing thresholds in the unaffected ear. After steroid treatment, less than half patients (47\%) showed hearing recovery. Simultaneous intratympanic dexamethasone (ITD) injections with systemic steroid did not confer an additional hearing gain or an earlier recovery rate in diabetic patients with SSNHL. In the multivariate analysis, initial hearing thresholds of affected ear and timing of steroid treatment were significantly associated with hearing prognosis in diabetic patients with SSNHL. Conclusions: Diabetic patients with SSNHL tended to have increased NLR, LMR, and PLR, which are reported to be associated with microvascular angiopathy. Simultaneous ITD injections to improve hearing recovery in diabetic patients with SSNHL seems unnecessary.

J Audiol Otol 2021;25(1):27-35

\section{Introduction}

Sudden sensorineural hearing loss (SSNHL) is defined as a sudden onset of sensorineural hearing loss of $30 \mathrm{~dB}$ or more over at least three contiguous audiometric frequencies [1]. Viral infections, vascular compromise, autoimmune processes, and labyrinthine membrane ruptures have been proposed as possible etiologies [1]. Although the exact mechanism of action of steroids is unclear, high dose steroid administration is the most commonly used treatment for SSNHL $[2,3]$. Intratympanic dexamethasone (ITD) injection is considered the treatment of choice for SSNHL patients with contraindica-

This is an Open Access article distributed under the terms of the Creative Commons Attribution Non-Commercial License (https://creativecommons.org/licenses/by-nc/4.0/) which permits unrestricted non-commercial use, distribution, and reproduction in any medium, provided the original work is properly cited. tions for systemic steroid or as salvage after systemic steroids [4].

With associated microangiopathy or inflammatory processes, diabetes mellitus (DM) could be a risk factor for SSNHL [5]. Additionally, microvascular problems in diabetic patients can affect the delivery and efficacy of systemic steroid in the cochlea [6,7]. Also, the use of systemic steroids can worsen glycemic control in diabetic patients. Given this, systemic steroid use for SSNHL in diabetic patients may lead to poorer outcomes than are observed in those without DM [8,9]. However, previous studies of SSNHL have seldom focused on diabetic patients.

Therefore, we conducted a retrospective review to evaluate the clinical factors associated with prognosis and compare the efficacy of simultaneous and sequential systemic steroid and ITD treatment in diabetic patients with SSNHL. Also, we 
evaluated the clinical features of SSNHL in diabetic patients including the laboratory data which are known to be related to systemic inflammation and glycemic control.

\section{Subjects and Methods}

\section{Subjects and design}

We retrospectively reviewed the medical records of all diabetic patients who admitted to Dankook University Hospital for SSNHL between January 2014 and July 2018. All patients in the present study exhibited unilateral SSNHL of at least $30 \mathrm{~dB}$ across at least three frequencies and occurring within 3 days of hearing loss. All patients received systemic high dose steroid therapy within one month after onset and had more than one month of follow-up audiogram. DM was defined as previously diagnosed type $2 \mathrm{DM}$ and the use of antidiabetic medications, such as oral antihyperglycemic agents or insulin. Exclusion criteria for the study included 1) a history of previous SSNHL; 2) bilateral SSNHL; 3) any recognized cause of SSNHL such as Meniere's disease, active viral infection, vestibular schwannoma, or congenital anomalies; 4) a history of chronic otitis media or otologic surgery; and 5) more than one month of the onset of the disease.

The basic characteristics of the patients, initial and followup audiograms, laboratory data, and methods of steroid treatment were collected. The basic characteristics included age, sex, side of affected ear, onset of symptoms, the presence of dizziness, and comorbid disease such as hypertension (HTN), cerebrovascular accident (CVA), and cardiovascular disease (CVD). Laboratory data, which included random plasma glucose, total cholesterol, glycosylated hemoglobin (HbA1c), and complete blood cell counts, were obtained before steroid treatment. The neutrophil-to-lymphocyte ratio (NLR), platelet-tolymphocyte ratio (PLR), and lymphocyte-to-monocyte ratio (LMR) were calculated as the ratio of neutrophil count to lymphocyte count, as the ratio of platelet count to lymphocyte count, and as the ratio of count lymphocyte to monocyte count, respectively. After steroid was administered, glucose stick test was daily performed to measure fasting glucose. Fasting glucose level in the morning (7 am) was collected to evaluate the glucose control during the high dose steroid treatment.

\section{Treatment protocol}

All patients received antiviral agent and systemic high dose steroid therapy (48 mg in the morning, $12 \mathrm{mg}$ in the evening, total $60 \mathrm{mg}$ of methylprednisolone for 7 days, followed by a taper for 7 days) within one month after onset. ITD injection was administered as a combination therapy along with the systemic high dose steroid or a salvage therapy for patients who did not exhibit recovery after an initial one week of systemic high dose steroid treatment. IT injections were performed 3-5 times with dexamethasone $(4 \mathrm{mg} / \mathrm{mL})$ within 2 weeks of diagnosis. The combination group defined as patients who received ITD injection along with the systemic high dose steroid. The non-combination group defined as patients who received only high dose steroid therapy or who received ITD injection as a salvage therapy after high dose steroid therapy. No additional treatment, including prostaglandin, vitamins, or hyperbaric oxygen therapy were used as second line treatments.

\section{Outcome evaluations and data analysis}

The initial audiogram was performed before receiving steroid treatment. Mean pure-tone thresholds were calculated at $0.5,1,2$, and $4 \mathrm{kHz}$ for air conduction thresholds. The degree of hearing loss was calculated as the difference of mean pure-tone thresholds between affected ear and unaffected ear for low frequencies $(0.25$ and $0.5 \mathrm{kHz})$, middle frequencies ( 1 and $2 \mathrm{kHz}$ ), and high frequencies ( 4 and $8 \mathrm{kHz}$ ). Hearing recovery was evaluated based on the result of the latest audiogram performed at least one month after treatment. The hearing gain was calculated as the difference between initial and final audiograms. Hearing improvement was assessed by modifying Siegel's criteria [10]. According to Siegel's criteria, complete or partial recovery was defined as patients whose final hearing level was better than $45 \mathrm{~dB} \mathrm{HL}$ and whose hearing gain was more than $15 \mathrm{~dB}$ HL. In this study, the hearing improved group was defined as patients whose degree of final hearing loss was less than $45 \mathrm{~dB} \mathrm{HL}$ and whose hearing gain was more than $15 \mathrm{~dB}$ HL.

\section{Statistical analysis}

The association of clinical or laboratory parameters with the hearing impairment were evaluated using Pearson's correlation, Spearman's correlation, independent t-test, and MannWhitney test. The differences between two different treatment groups were analyzed using independent t-test, chi-square test, and Mann-Whitney test. To investigate the clinical factors related to hearing recovery, independent t-test, Mann-Whitney test, and chi-square test were used. Multivariate logistic regression model was performed to assess the independent association of treatment method and hearing recovery. Clinical factors found to have possible association in univariate analysis $(p<$ 0.20 ) were entered into the multivariate logistic regression analysis model. All statistical analyses were performed using SPSS software (ver. 18.0, SPSS Inc., Chicago, IL, USA) and $p$ values less than 0.05 were considered statistically significant. 


\section{Ethics statement}

This retrospective study protocol was approved by the Dankook University Hospital Institutional Review Board (IRB No. 2019-12-008), and it was performed in accordance with relevant guidelines and regulations. All data were fully anonymized before analyzing and the Institutional Review Board waived the requirement for informed consent.

\section{Results}

\section{Demographics and clinical characteristics}

Of 77 patients with unilateral SSNHL and DM, 47 patients were followed more than one month. Finally, 47 patients (25 male and 22 female) with a mean age of $58 \pm 14$ (range $31-$ 82 ) years were included in this study. Clinical characteristics are shown in Table 1. The mean random plasma glucose and $\mathrm{HbAlc}$ at admission were $217 \pm 92 \mathrm{mmol} / \mathrm{L}$ and $8.2 \pm 1.6 \%$, respectively. About half of the patients (22/47) had a random plasma glucose lower than $200 \mathrm{mg} / \mathrm{dL}$ and only $26 \%$ (12/47) of the patients had a HbA1c lower than $7.0 \%$. Thirty-one $(65.9 \%)$

Table 1. Clinical characteristics of the study population $(n=47)$

\begin{tabular}{|c|c|}
\hline Variables & \\
\hline Age (years) & $58 \pm 14$ \\
\hline Sex (male:female) & $25: 22$ \\
\hline Side (right:left) & $15: 32$ \\
\hline Onset of symptoms (days) & $4.8 \pm 6.3$ \\
\hline Dizziness (no:yes) & $32: 15$ \\
\hline Comorbid disease (no:yes) & $16: 31$ \\
\hline \multicolumn{2}{|l|}{ Audiogram of affected ear } \\
\hline Initial PTA (4 FA, dB HL) & $85.3 \pm 20.3$ \\
\hline Initial word recognition scores (\%) & $12.4 \pm 25.8$ \\
\hline \multicolumn{2}{|l|}{ Laboratory data } \\
\hline Random plasma glucose (mg/dL) & $217 \pm 92$ \\
\hline Glycosylated hemoglobin (\%) & $8.2 \pm 1.6$ \\
\hline Total cholesterol (mg/dL) & $172 \pm 32$ \\
\hline Neutrophil-to-lymphocyte ratio & $4.49 \pm 3.25$ \\
\hline Platelet-to-lymphocyte ratio & $158.7 \pm 83.5$ \\
\hline Lymphocyte-to-monocyte ratio & $10.34 \pm 17.16$ \\
\hline $\begin{array}{l}\text { Fasting glucose level during steroid treatment } \\
(\mathrm{mg} / \mathrm{dL})\end{array}$ & $216 \pm 92$ \\
\hline \multicolumn{2}{|l|}{ Steroid treatment } \\
\hline Systemic steroid only & 16 \\
\hline Systemic steroid+IT injections (sequential) & 6 \\
\hline Systemic steroid+IT injections (simultaneous) & 25 \\
\hline Duration of follow-up (days) & $82 \pm 35$ \\
\hline
\end{tabular}

Continuous variables are presented with mean \pm standard deviation or $\mathrm{n}$. Comorbid disease included hypertension, cerebrovascular accident, and cardiovascular disease. Mean PTA was calculated for 4 FA. PTA: pure-tone average, 4 FA: four frequencies $(0.5,1,2$, and $4 \mathrm{kHz}), \mathrm{IT}$ : intratympanic patients had comorbid disease such as HTN, CVA, and CVD. In laboratory data at admission, mean cholesterol level was $172 \pm 32 \mathrm{mg} / \mathrm{dL}$. Most of the patients (33/47) had cholesterol levels lower than $200 \mathrm{mg} / \mathrm{dL}$. The mean NLR, PLR, and LMR were $4.49 \pm 3.25,158.7 \pm 83.5$, and $10.34 \pm 17.16$, respectively. Compared to reference values in healthy subjects $(1.65$ for NLR, 132.4 for PLR, and 5.31 for LMR), 79\% (37/47), $55 \%(26 / 47)$, and $45 \%(21 / 47)$ of the patients had higher values of mean NLR, PLR, and LMR, respectively [11].

Fifteen $(31.9 \%)$ patients had unilateral SSNHL in the right ears, whereas $32(68.1 \%)$ patients had left ear involvement. On the initial audiogram of affected ear, the mean pure tone threshold was $85.3 \pm 20.3 \mathrm{~dB}$ HL and the mean word recognition score (WRS) was $12.4 \pm 25.8 \%$. Fifteen patients $(31.9 \%)$ presented with associated dizziness. All patients received high dose steroid treatment on average $4.8 \pm 6.3$ days after onset. Among them, 25 patients (53.2\%) received ITD injections as a combination therapy, 6 patients $(12.8 \%)$ received ITD injections as a salvage therapy, and 16 patients (34\%) received no ITD injections. During the systemic steroid, mean fasting glucose level in the morning was $216 \pm 92 \mathrm{mg} / \mathrm{dL}$. The mean follow-up period was $54 \pm 38$ days.

\section{Degree of hearing loss in DM patients}

Table 2 shows the associations of clinical or laboratory parameters with the hearing impairment after SSNHL. The degree of hearing loss in low, middle, and high frequencies were $61.6 \pm 20.5,66.4 \pm 22.9$, and $45.5 \pm 25.8$, respectively. Older diabetics showed significant hearing loss of the unaffected ear, but they did not show a significant correlation with hearing loss of the affected ear. Increased age had significant negative association with the degree of hearing loss in middle and high frequencies ( $r=-0.300, p=0.041$ for middle frequencies and $r=-0.429, p=0.003$ for high frequencies). Male had significant hearing loss at high frequencies of the unaffected ear compared to female, but there was no significant association with hearing loss of the affected ear or the degree of hearing loss. The presence of dizziness or comorbid disease showed significant association with hearing loss at middle frequencies of the unaffected ear, but it did not show a significant association with the degree of hearing loss. Laboratory parameters showed no significant correlation with the degree of hearing loss. Only $\mathrm{HbA1C}$ and total cholesterol had a significant negative correlation with hearing loss at high frequencies in the affected ear $(\mathrm{r}=-0.321, p=0.041$ for HbA1C) and unaffected ear $(\mathrm{r}=$ $-0.324, p=0.037$ for total cholesterol), respectively. 
Diabetic Patients with SSNHL

Table 2. Associations of clinical and laboratory parameters with the hearing impairment

\begin{tabular}{|c|c|c|c|c|c|c|c|c|c|}
\hline \multirow{2}{*}{ Variables } & \multicolumn{3}{|c|}{ Unaffected ear } & \multicolumn{3}{|c|}{ Affected ear } & \multicolumn{3}{|c|}{ Degree of hearing loss } \\
\hline & LT & MT & HT & LT & MT & HT & LT & MT & HT \\
\hline Pure tone thresholds ( $\mathrm{dB} \mathrm{HL})$ & $18 \pm 12$ & $20 \pm 13$ & $38 \pm 21$ & $79 \pm 21$ & $86 \pm 22$ & $83 \pm 17$ & $62 \pm 21$ & $66 \pm 23$ & $46 \pm 26$ \\
\hline \multicolumn{10}{|l|}{ Age } \\
\hline r & 0.502 & 0.642 & 0.599 & 0.193 & 0.054 & 0.079 & -0.105 & -0.300 & -0.429 \\
\hline$p$-value & $<0.001^{*}$ & $<0.001^{*}$ & $<0.001^{*}$ & $0.194^{*}$ & $0.720^{*}$ & $0.599 *$ & $0.484^{*}$ & $0.041^{*}$ & $0.003^{*}$ \\
\hline \multicolumn{10}{|l|}{ Sex } \\
\hline Male & $17 \pm 10$ & $19 \pm 11$ & $44 \pm 20$ & $76 \pm 24$ & $84 \pm 23$ & $84 \pm 15$ & $59 \pm 22$ & $65 \pm 22$ & $40 \pm 23$ \\
\hline Female & $19 \pm 15$ & $21 \pm 14$ & $31 \pm 20$ & $83 \pm 16$ & $89 \pm 21$ & $83 \pm 19$ & $65 \pm 18$ & $68 \pm 24$ & $52 \pm 27$ \\
\hline$p$-value & $0.923^{\S}$ & $0.772^{\S}$ & $0.023^{\dagger}$ & $0.416^{\S}$ & $0.471^{\S}$ & $0.845^{\S}$ & $0.411^{\S}$ & $0.597^{+}$ & $0.087^{+}$ \\
\hline \multicolumn{10}{|l|}{ Dizziness } \\
\hline No & $16 \pm 10$ & $16 \pm 10$ & $37 \pm 20$ & $75 \pm 22$ & $83 \pm 22$ & $81 \pm 19$ & $59 \pm 21$ & $67 \pm 23$ & $45 \pm 26$ \\
\hline Yes & $21 \pm 16$ & $27 \pm 15$ & $41 \pm 22$ & $88 \pm 16$ & $81 \pm 19$ & $88 \pm 12$ & $67 \pm 19$ & $66 \pm 23$ & $47 \pm 26$ \\
\hline$p$-value & $0.218^{\S}$ & $0.011^{\S}$ & $0.516^{*}$ & $0.039^{\S}$ & $0.168^{\dagger}$ & $0.296^{\S}$ & $0.250^{中}$ & $0.938^{*}$ & $0.747^{\dagger}$ \\
\hline \multicolumn{10}{|l|}{ Comorbid disease } \\
\hline No & $12 \pm 5$ & $13 \pm 8$ & $33 \pm 22$ & $78 \pm 19$ & $86 \pm 19$ & $86 \pm 14$ & $66 \pm 18$ & $73 \pm 23$ & $53 \pm 27$ \\
\hline Yes & $21 \pm 14$ & $23 \pm 13$ & $41 \pm 20$ & $80 \pm 22$ & $86 \pm 24$ & $82 \pm 19$ & $59 \pm 22$ & $63 \pm 23$ & $42 \pm 25$ \\
\hline$p$-value & $0.011^{\S}$ & $0.005^{\S}$ & $0.516^{*}$ & $0.597^{\S}$ & $0.168^{4}$ & $0.715^{\S}$ & $0.250^{\dagger}$ & $0.938^{4}$ & $0.747^{\dagger}$ \\
\hline \multicolumn{10}{|l|}{ Glucose (mg/dL) } \\
\hline r & 0.181 & 0.191 & -0.020 & -0.135 & -0.077 & -0.149 & -0.247 & -0.179 & -0.083 \\
\hline p-value & $0.223^{*}$ & $0.198^{*}$ & $0.893^{*}$ & $0.366^{*}$ & $0.605^{*}$ & $0.317^{*}$ & $0.094^{*}$ & $0.229 *$ & $0.579 *$ \\
\hline \multicolumn{10}{|l|}{ Glycosylated hemoglobin (\%) } \\
\hline r & -0.171 & 0.063 & -0.177 & -0.236 & -0.252 & -0.321 & -0.104 & -0.140 & 0.005 \\
\hline$p$-value & $0.286^{\dagger}$ & $0.698^{\dagger}$ & $0.267^{*}$ & $0.137^{\dagger}$ & $0.112^{\dagger}$ & $0.041^{\dagger}$ & $0.517^{*}$ & $0.381^{*}$ & $0.973^{*}$ \\
\hline \multicolumn{10}{|l|}{ Total cholesterol (mg/dL) } \\
\hline r & -0.217 & -0.254 & -0.324 & 0.028 & -0.092 & -0.072 & 0.159 & 0.053 & 0.218 \\
\hline$p$-value & $0.167^{*}$ & $0.105^{*}$ & $0.037^{*}$ & $0.860^{*}$ & $0.563^{*}$ & $0.649 *$ & $0.314^{*}$ & $0.738^{*}$ & $0.166^{*}$ \\
\hline \multicolumn{10}{|l|}{ Neutrophil-to-lymphocyte ratio } \\
\hline r & -0.116 & -0.049 & 0.114 & -0.188 & -0.265 & -0.224 & -0.264 & -0.151 & -0.286 \\
\hline p-value & $0.436^{\dagger}$ & $0.743^{\dagger}$ & $0.444^{*}$ & $0.206^{\dagger}$ & $0.072^{\dagger}$ & $0.130^{\dagger}$ & $0.073^{*}$ & $0.312^{*}$ & $0.052^{*}$ \\
\hline \multicolumn{10}{|l|}{ Platelet-to-lymphocyte ratio } \\
\hline r & -0.192 & -0.173 & -0.061 & -0.109 & -0.086 & -0.133 & 0.003 & 0.013 & -0.040 \\
\hline$p$-value & $0.197^{*}$ & $0.244^{*}$ & $0.685^{*}$ & $0.464^{*}$ & $0.566^{*}$ & $0.373^{*}$ & $0.983^{*}$ & $0.934^{*}$ & $0.791^{*}$ \\
\hline \multicolumn{10}{|c|}{ Lymphocyte-to-monocyte ratio } \\
\hline r & -0.025 & 0.143 & 0.006 & -0.220 & -0.155 & -0.141 & -0.013 & -0.034 & -0.006 \\
\hline$p$-value & $0.867^{\dagger}$ & $0.338^{\dagger}$ & $0.968^{*}$ & $0.137^{\dagger}$ & $0.300^{\dagger}$ & $0.344^{\dagger}$ & $0.929 *$ & $0.821^{*}$ & $0.968^{*}$ \\
\hline
\end{tabular}

Continuous variables are presented with mean \pm standard deviation. *pearson's correlation analysis, ${ }^{\dagger}$ spearman correlation analysis, "independent t-test, ${ }^{\S}$ Mann-Whitney test. LT: low tone $(0.25$ and $0.5 \mathrm{kHz}), \mathrm{MT}$ : middle tone ( 1 and $\left.2 \mathrm{kHz}\right)$, HT: high tone (4 and 8 $\mathrm{kHz})$.

\section{Hearing outcome according to method of steroid treatment}

Twenty-five patients who received ITD injections as a combination therapy belonged to the combination group, and 22 patients who received no ITD injection or ITD injections as a salvage therapy belonged to the non-combination group. Two groups showed no statistically significant differences in age, sex, side of the lesion, onset of symptoms, presence of dizziness, comorbid disease, and duration of follow-up (Table 3). Initial audiogram including pure tone average, WRS, and the degree of hearing loss showed no significant difference between the two groups. Laboratory data also showed no significant differences between the two groups in random plasma glucose, $\mathrm{HbAlc}$, total cholesterol, NLR, PLR, LMR, and fasting glucose level during steroid treatment.

Fig. 1 shows the hearing outcome in combination group (Fig. 1A and B) and non-combination group (Fig. 1C and D). Pure tone thresholds and WRSs were presented at three different time points (baseline, one week after treatment, and last follow-up). There was a statistically significant improve- 
Ju YR, et al.

Table 3. Comparison of demographic and clinical parameters according to method of steroid treatment

\begin{tabular}{|c|c|c|c|}
\hline Variables & Combination group & Non-combination group & p-values \\
\hline Number of patients & 25 & 22 & \\
\hline Age (years) & $59.8(31-82)$ & $58.0(31-76)$ & $0.620^{*}$ \\
\hline Sex (male:female) & $14: 11$ & $11: 11$ & $0.681^{\dagger}$ \\
\hline Side lesion (right:left) & $10: 15$ & $5: 17$ & $0.205^{\dagger}$ \\
\hline Onset of symptoms (days) & $4.81 \pm 5.48$ & $4.60 \pm 6.17$ & $0.879^{\dagger}$ \\
\hline Dizziness (no:yes) & $15: 10$ & $17: 5$ & $0.205^{\dagger}$ \\
\hline Comorbid disease (no:yes) & $7: 18$ & $9: 13$ & $0.351^{\dagger}$ \\
\hline \multicolumn{4}{|l|}{ Audiogram } \\
\hline Initial pure-tone average (4 FA, dB HL) & $82.3 \pm 20.2$ & $85.1 \pm 20.4$ & $0.758^{*}$ \\
\hline Initial word recognition scores (\%) & $13.2 \pm 25.9$ & $12.6 \pm 26.0$ & $0.268^{\dagger}$ \\
\hline Degree of hearing loss ( $4 \mathrm{FA}, \mathrm{dB} \mathrm{HL}$ ) & $58.5 \pm 19.4$ & $62.8 \pm 21.4$ & $0.547^{*}$ \\
\hline \multicolumn{4}{|l|}{ Laboratory data } \\
\hline Random plasma glucose (mg/dL) & $209.2 \pm 96.8$ & $224.8 \pm 87.4$ & $0.567^{*}$ \\
\hline Hemoglobin Alc (\%) & $8.04 \pm 1.35$ & $8.19 \pm 1.64$ & $0.234^{\dagger}$ \\
\hline Total cholesterol (mg/dL) & $172.2 \pm 31.3$ & $172.3 \pm 32.3$ & $0.602^{*}$ \\
\hline Neutrophil-to-lymphocyte ratio & $4.77 \pm 3.32$ & $4.46 \pm 3.28$ & $0.587^{*}$ \\
\hline Platelet-to-lymphocyte ratio & $156.7 \pm 73.2$ & $157.9 \pm 84.2$ & $0.908^{*}$ \\
\hline Lymphocyte-to-monocyte ratio & $9.8 \pm 16.1$ & $10.4 \pm 17.3$ & $0.394^{\dagger}$ \\
\hline Fasting glucose level during steroid treatment (mg/dL) & $179.3 \pm 48.7$ & $188.0 \pm 50.9$ & $0.382^{\dagger}$ \\
\hline Duration of follow-up (days) & $72 \pm 32$ & $94 \pm 35$ & $0.432 *$ \\
\hline
\end{tabular}

Continuous variables are presented with mean \pm standard deviation or $n .{ }^{*}$ independent t-test, ${ }^{\dagger}$ Mann-Whitney test, ${ }^{*}$ chi-square test. $4 \mathrm{FA}$ : four frequencies $(0.5,1,2$, and $4 \mathrm{kHz})$

ment in pure tone thresholds and WRSs after steroid treatment in combination group $\left[\chi^{2}(3)=24.89, p<0.001\right.$ for $0.25 \mathrm{kHz}$, $\chi^{2}(3)=26.9, p<0.001$ for $0.5 \mathrm{kHz}, \chi^{2}(3)=28.62, p<0.001$ for $1 \mathrm{kHz}, \chi^{2}(3)=28.62, p<0.001$ for $2 \mathrm{kHz}, \chi^{2}(3)=21.27$, $p<0.001$ for $4 \mathrm{kHz}, \chi^{2}(3)=9.088, p=0.011$ for $8 \mathrm{kHz}$, and $\chi^{2}(3)=28.64, p<0.001$ for WRS] and non-combination group $\left[\chi^{2}(3)=33.56, p<0.001\right.$ for $0.25 \mathrm{kHz}, \chi^{2}(3)=29.54, p<0.001$ for $0.5 \mathrm{kHz}, \chi^{2}(3)=30.6, p<0.001$ for $1 \mathrm{kHz}, \chi^{2}(3)=26.95$, $p<0.001$ for $2 \mathrm{kHz}, \chi^{2}(3)=17.45, p<0.001$ for $4 \mathrm{kHz}, \chi^{2}(3)=9$, $p=0.011$ for $8 \mathrm{kHz}$, and $\chi^{2}(3)=23.84, p<0.001$ for WRS]. Post hoc analysis with Dunn's multiple comparison test was conducted to compare the pure tone thresholds and WRSs at baseline with the hearing outcome at one week after treatment and last follow-up. Although there were no significant differences between baseline and one week after treatment in both groups, there were significant improvements in pure tone thresholds at all frequencies and WRS in both groups (all $p$ values $<0.05$ ).

Fig. 2 shows a comparison of hearing improvement in pure tone thresholds and WRSs between combination group and non-combination group. The non-combination group showed a slight better hearing improvement in pure tone thresholds and in WRSs, but which was not statistically significant. The combination group showed the highest hearing improvement in $500 \mathrm{~Hz}$ and the lowest in $8 \mathrm{kHz}$.

\section{Clinical factors related to hearing recovery}

Among 47 patients, 25 patients were hearing non-improved group, and 22 patients were improved group. Table 4 shows the clinical factors related to hearing recovery. Age, comorbid dizziness, initial PTA and WRS of ipsilateral ear were significantly associated with hearing recovery (all $p<0.05$ ). A multivariate logistic regression was performed to ascertain the effects of clinical factors on the likelihood of hearing recovery. Variables (age, sex, onset of symptom, dizziness, initial PTA, initial WRS, and duration of follow-up) found to have possible association in univariate analysis $(p<0.20)$ were entered into the multivariate logistic regression analysis model. However, age, sex, initial WRS, and duration of follow-up were removed in multivariate logistic regression model with backward selection. The final logistic regression model with backward selection was statistically significant, $\chi^{2}(3)=22.259, p<0.001$. The model explained $50.4 \%$ (Nagelkerke $\mathrm{R}^{2}$ ) of the variance in hearing recovery and correctly classified $78.7 \%$ of cases. Longer duration of onset was associated with 0.720 times decreased likelihood of hearing recovery, and worse initial PTA was associated with 0.938 times decreased likelihood of hearing recovery. 

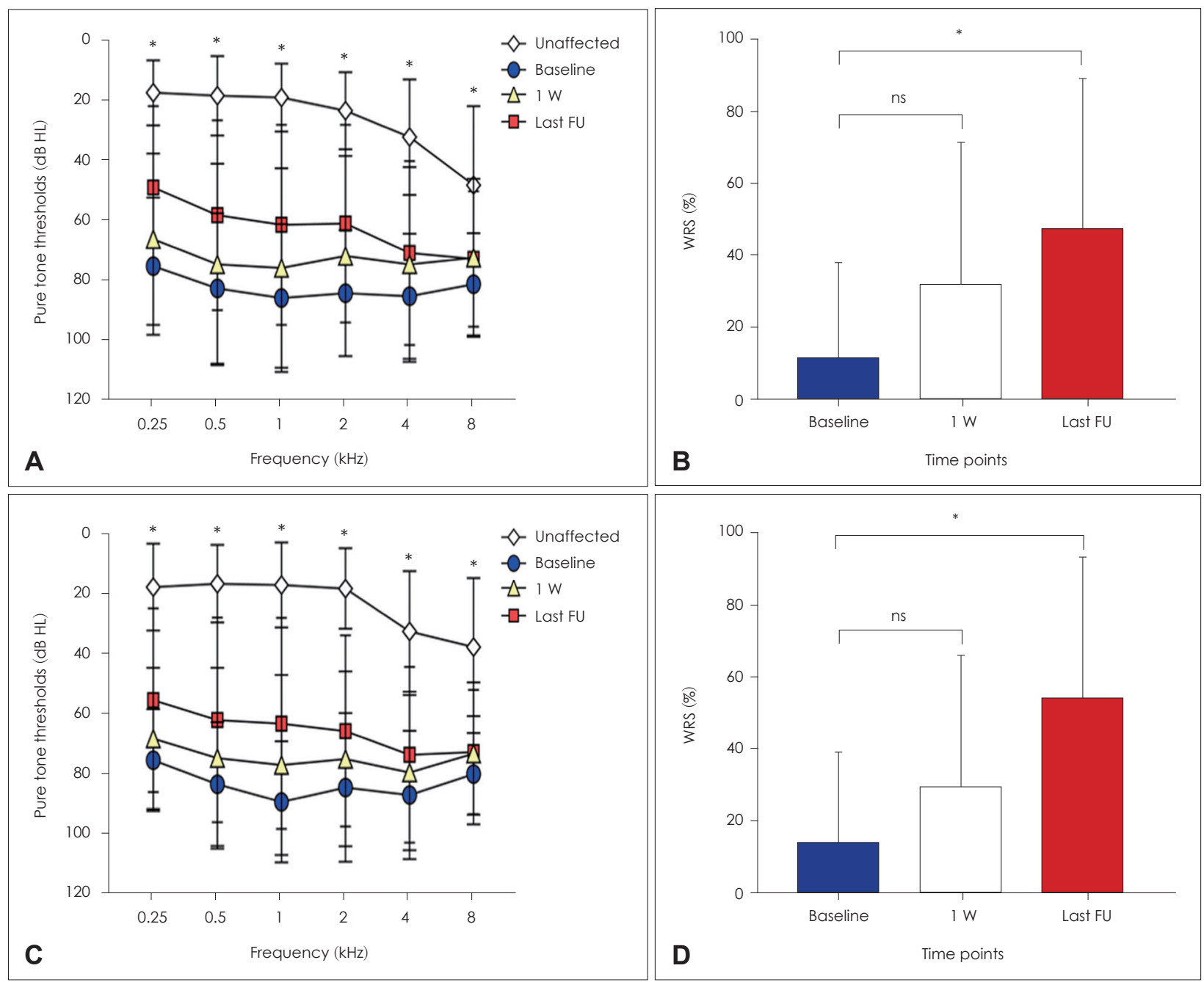

Fig. 1. Hearing outcome in combination and non-combination groups. Fig. 1 shows the results of PTA and WRS in combination group (A and $B$ ) and non-combination group (C and D). Pure tone thresholds and WRSs were presented at three different time points (baseline, one week after treatment, and last follow-up). "post hoc analysis between baseline and last follow-up. PTA: pure-tone average, WRS: word recognition score.

\section{Discussion}

DM is one of the most common metabolic disorders, which is associated with hearing impairment [12,13]. Previous studies demonstrated that poorly controlled diabetics have significant hearing loss in high frequencies as compared with well controlled diabetes $[12,14,15]$. There is still a deal of controversy but, angiopathy and neuropathy have been considered important factors for the vestibular-cochlear disorders found in diabetic patients [16]. Currently, histopathological findings supported that the increase in hearing threshold is attributed to microvascular angiopathy occurring in capillaries of stria vascularis which make these vessels thicker than normal $[17,18]$. Many studies have been done to find out the association between glucose level and hearing loss. However, little has been reported about the factors responsible for worsening hearing thresholds or prognosis of hearing recovery in diabetics with SSNHL.

Our data clearly revealed hearing deficits of unaffected ear with increasing age in all frequencies (Table 2). Age was not correlated with the hearing thresholds of affected ear, but it had a significant negative correlation with the degree of hearing loss in the middle and high frequencies. This means that in older patients, the threshold difference between the affected and unaffected ears was small. Possibly, this is because baseline hearing impairment in older diabetics are common, thus further hearing loss due to SSNHL could relatively be less prominent. The other clinical factors were not significantly correlated with the degree of hearing loss (Table 2). As in the general population [19], males had significantly worse hearing thresholds in the high frequencies of the unaffected ear compared to females. Diabetics who had dizziness or comor- 
bid disease such as HTN, CVA, and CVD showed significantly worse hearing thresholds in low, middle, and high frequencies of the unaffected ear.
Previous study reported that a high postprandial plasma glucose level had a significant negative correlation with the degree of hearing and a positive correlation with the baseline hearing

Fig. 2. Hearing improvement between combination and non-combination groups. Fig. 2 shows a comparison of hearing improvement in pure tone thresholds and WRSs between combination group and noncombination group. The non-combination group showed a slight better hearing improvement in pure tone thresholds and WRSs, but which was not statistically significant. Avg: average, WRS: word recognition score.

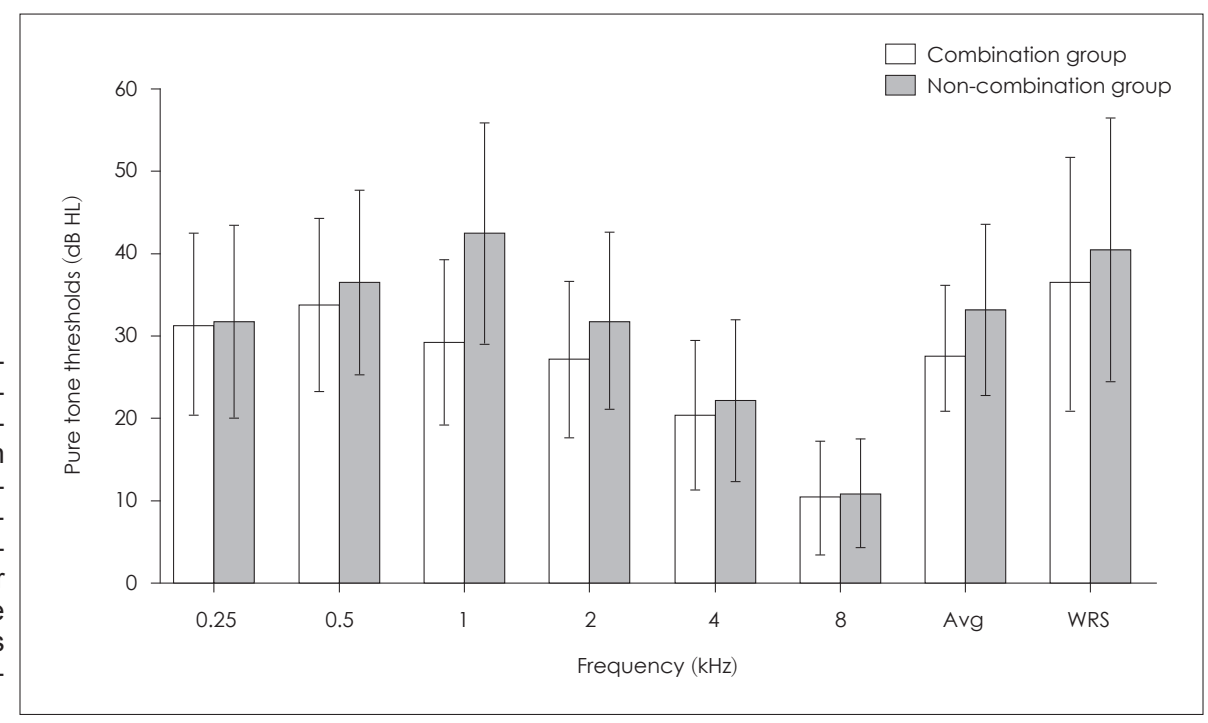

Table 4. Clinical factors related to hearing recovery

\begin{tabular}{|c|c|c|c|c|c|c|c|c|}
\hline \multirow{2}{*}{ Variables } & \multirow{2}{*}{$\begin{array}{l}\text { Non-improved } \\
\quad(n=25)\end{array}$} & \multirow{2}{*}{$\begin{array}{l}\text { Improved } \\
(\mathrm{n}=22)\end{array}$} & \multirow{2}{*}{$\begin{array}{l}\text { Univariate } \\
\text { p-value }\end{array}$} & \multicolumn{5}{|c|}{ Multivariate ${ }^{\S}$} \\
\hline & & & & B & SE & OR & $95 \% \mathrm{Cl}$ & p-value \\
\hline Age (years) & $62 \pm 13$ & $53 \pm 13$ & $0.021^{*}$ & & & & & \\
\hline Sex (male:female) & $11: 14$ & $14: 8$ & $0.178^{4}$ & & & & & \\
\hline Side lesion (right:left) & $7: 18$ & $8: 14$ & $0.539^{\dagger}$ & & & & & \\
\hline Onset of symptoms (days) & $4.8 \pm 6.3$ & $4.6 \pm 6.3$ & $0.116^{\dagger}$ & -0.328 & 0.159 & 0.720 & $0.527-0.984$ & 0.039 \\
\hline Dizziness (no:yes) & $13: 12$ & $19: 3$ & $0.012^{\dagger}$ & -1.689 & 0.866 & 0.185 & $0.034-1.007$ & 0.051 \\
\hline Comorbid disease (no:yes) & $8: 17$ & $8: 14$ & $0.753^{*}$ & & & & & \\
\hline \multicolumn{9}{|l|}{ Initial PTA (4 FA, dB HL) } \\
\hline Unaffected ear & $24.3 \pm 12.6$ & $20.1 \pm 12.1$ & $0.232^{\dagger}$ & & & & & \\
\hline Affected ear & $92.0 \pm 18.8$ & $77.8 \pm 19.6$ & $0.015^{\dagger}$ & -0.064 & 0.025 & 0.938 & $0.893-0.985$ & 0.010 \\
\hline \multicolumn{9}{|l|}{ Initial WRS (\%) } \\
\hline Unaffected ear & $95.0 \pm 12.9$ & $96.7 \pm 6.1$ & $0.540^{\dagger}$ & & & & & \\
\hline Affected ear & $5.6 \pm 17.3$ & $20.1 \pm 31.7$ & $0.049^{\dagger}$ & & & & & \\
\hline \multicolumn{9}{|l|}{ Laboratory data } \\
\hline Random plasma glucose (mg/dL) & $206 \pm 86$ & $228 \pm 99$ & $0.431^{*}$ & & & & & \\
\hline Glycosylated hemoglobin (\%) & $8.03 \pm 1.68$ & $8.33 \pm 1.61$ & $0.548^{\dagger}$ & & & & & \\
\hline Total cholesterol (mg/dL) & $169 \pm 32$ & $175 \pm 32$ & $0.535^{*}$ & & & & & \\
\hline Neutrophil-to-lymphocyte ratio & $4.33 \pm 2.65$ & $4.67 \pm 3.88$ & $0.639^{\dagger}$ & & & & & \\
\hline Platelet-to-lymphocyte ratio & $149 \pm 56$ & $169 \pm 107$ & $0.435^{*}$ & & & & & \\
\hline Lymphocyte-to-monocyte ratio & $10.5 \pm 18.7$ & $10.2 \pm 15.7$ & $0.701^{\dagger}$ & & & & & \\
\hline $\begin{array}{l}\text { Fasting glucose during steroid } \\
\text { treatment (mg/dL) }\end{array}$ & $182 \pm 50$ & $194 \pm 51$ & $0.413^{*}$ & & & & & \\
\hline Duration of follow-up (days) & $63 \pm 44$ & $44 \pm 28$ & $0.071^{*}$ & & & & & \\
\hline $\begin{array}{l}\text { Steroid treatment } \\
\text { (combination:non-combination) }\end{array}$ & $14: 11$ & $11: 11$ & $0.681^{*}$ & & & & & \\
\hline
\end{tabular}

Continuous variables are presented with mean \pm standard deviation or $n$. Variables (age, sex, onset of symptom, dizziness, initial PTA, initial WRS, and duration of follow-up) found to have possible association in univariate analysis $(p<0.20)$ were entered into the multivariate logistic regression analysis model. *independent t-test, ${ }^{\dagger}$ Mann-Whitney test, ${ }^{\dagger}$ chi-square test, ${ }^{\S}$ logistic regression test. 4 FA: four frequencies $(0.5,1,2$, and $4 \mathrm{kHz})$, PTA: pure-tone average, WRS: word recognition score, SE: standard error, OR: odds ratio, $\mathrm{Cl}$ : confidence interval 
thresholds loss in middle frequencies [20]. However, random plasma glucose and $\mathrm{HbA1c}$ levels were not significantly correlated with the hearing thresholds of unaffected ear, as well as the degree of hearing loss. The mean values of NLR, LMR, and PLR in diabetics with SSNHL were increased compared to healthy adult in South Korea (Table 1). These indicators, which are known to be related to systemic inflammation, have been reported to be associated with microvascular angiopathy in patients with SSNHL [21] or diabetic patients [22,23]. Chronic inflammations can cause microvascular injury [24]. Although there was no significant correlation between these indicators and hearing deficits (Table 2), increased NLR, LMR, and PLR suggested that microangiopathy may be important pathogenesis of SSNHL in diabetics patients.

Systemic steroids are widely used for SSNHL, but its effectiveness may be decreased by their limited permeability through the blood-perilymphatic barrier [25]. Furthermore, diabetic angiopathies in the blood vessels supplying the inner ear may cause reduction of microcirculation in the cochlea. Several studies of animal and human temporal bones revealed thickened and damaged capillary walls of the stria vascularis with diabetes [6,8]. Previously, Ahn, et al. [26] reported that the hearing recovery of SSNHL after high dose steroid treatment was only $38 \%$ (12/32) in diabetics, while 58\% (60/103) in nondiabetics. ITD injections directly affect inner ear function via diffusion through the round window, resulting in increased perilymph steroid concentrations [8,25]. Given these findings, we assumed that simultaneous ITD injections with systemic steroid treatment may confer an additional hearing gain or an earlier recovery rate compared to salvage ITD injections following systemic steroids. In our study, all diabetics patients received high dose systemic steroid treatment and/or ITD injections. Although standard ITD regimens, including the timing and frequency of ITD injections have not been developed as salvage treatment, many studies reported better results when salvage treatment was started within 2 weeks from the onset $[27,28]$. Thus, non-combination group received salvage treatment after one week after initial treatment failure. The overall rate of hearing recovery after steroid treatment was $46 \%(22 / 47)$ in diabetics. However, our data showed no superiority of combination therapy over non-combination therapy (Figs. 1 and 2). Although combination and non-combination groups showed significant hearing recovery at least one month after treatment (Fig. 1), non-combination group showed a slight better hearing improvement than combination group, which was not statistically significant (Fig. 2).

The factors responsible which influence the hearing recovery in diabetics were evaluated. In univariate analysis, age, the presence of dizziness, initial pure tone thresholds of affected ear were significantly associated with hearing recovery (Table 4). In multivariate analysis, diabetic patients with early steroid treatment had an increased likelihood of hearing recovery, and diabetics with worse initial hearing thresholds had a decreased likelihood of hearing recovery. Diabetic patients with concurrent dizziness also had a decreased likelihood of hearing recovery, but which was not statistically significant. Because these patients had worse hearing thresholds compared to diabetics without dizziness (Table 2), initial hearing thresholds seems more relevant to hearing recovery than the dizziness. Method of treatment, glycemic controls (random plasma glucose, HbAlc, and fasting glucose during steroid treatment) or systemic inflammation level (NLR, PLR, and LMR) were not significantly associated with hearing recovery. Previous studies reported that combination steroid therapy did not show superiority of hearing recovery in patients with SSNHL [29], even for severe-to-profound SSNHL [30]. Considering ITD injections can be decided upon by the status of hearing improvement after an oral steroid as salvage regimen, even in patients who were expected to have a poor prognosis.

There are several limitations in our study. The first, since this was a retrospective study, some diabetic patients were dropped out during follow-up periods. Second, the sample size was small to generalize. Third, the results may have been influenced by cotreatment with ginko biloba. We prescribed a ginko biloba inconsistently depending on the history of taking anticoagulant or antiplatelet drugs. Forth, chronic complications of diabetes such as nephropathy, retinopathy, and neuropathy were not recorded in detail. In our data, all diabetic patients with comorbid dizziness had HTN. Thus, comorbid disease could not reflect the severity of diabetic complications.

In summary, diabetic patients with SSNHL tended to have increased NLR, LMR, and PLR, which are reported to be associated with microvascular angiopathy. Baseline hearing thresholds were worse in older diabetics or diabetics with dizziness and hypertension. However, there was no clinical factor or laboratory parameter associated with the degree of hearing loss. After steroid treatment, less than half patients (47\%) showed hearing recovery. Simultaneous ITD injections with systemic steroid did not confer an additional hearing gain or an earlier recovery rate. Initial hearing thresholds and timing of steroid treatment were significantly associated with hearing prognosis in diabetic patients with SSNHL.

\section{Acknowledgments}

None

\section{Conflicts of interest}

The authors have no financial conflicts of interest. 


\section{Author Contributions}

Conceptualization: Ji Eun Choi. Data curation: Yeo Rim Ju. Formal analysis: Ji Eun Choi. Methodology: Yeo Rim Ju and Ji Eun Choi. Project administration: Min Young Lee and Jae Yun Jung. Resources: Ji Eun Choi, Min Young Lee, and Jae Yun Jung. Software: Yeo Rim Ju and Ji Eun Choi. Supervision: Ji Eun Choi and Jae Yun Jung. Validation: Min Youn Lee and Ji Eun Choi. Visualization: Ji Eun Choi. Writing — original drift: Yeo Rim Ju and Ji Eun Choi. Writing — review \& editing: Yeo Rim Ju and Ji Eun Choi. Approval of final manuscript: all authors.

\section{ORCID iDs}

Yeo Rim Ju

Hyoung-sik Park

Min Young Lee

Jae Yun Jung

Ji Eun Choi https://orcid.org/0000-0003-1754-9916

https://orcid.org/0000-0002-7160-865X

https://orcid.org/0000-0002-6860-8042

https://orcid.org/0000-0002-1870-0748

https://orcid.org/0000-0001-8105-813X

\section{REFERENCES}

1) Hughes GB, Freedman MA, Haberkamp TJ, Guay ME. Sudden sensorineural hearing loss. Otolaryngol Clin North Am 1996;29:393405.

2) Wei BP, Stathopoulos D, O'Leary S. Steroids for idiopathic sudden sensorineural hearing loss. Cochrane Database Syst Rev 2013;2013: CD003998.

3) Battaglia A, Burchette R, Cueva R. Combination therapy (intratympanic dexamethasone+high-dose prednisone taper) for the treatment of idiopathic sudden sensorineural hearing loss. Otol Neurotol 2008; 29:453-60.

4) Wu HP, Chou YF, Yu SH, Wang CP, Hsu CJ, Chen PR. Intratympanic steroid injections as a salvage treatment for sudden sensorineural hearing loss: a randomized, double-blind, placebo-controlled study. Otol Neurotol 2011;32:774-9.

5) Cameron NE, Eaton SE, Cotter MA, Tesfaye S. Vascular factors and metabolic interactions in the pathogenesis of diabetic neuropathy. Diabetologia 2001;44:1973-88.

6) Jorgensen MB. The inner ear in diabetes mellitus. Histological studies. Arch Otolaryngol 1961;74:373-81.

7) Kim HJ, Lee YJ, Kang HT, Lee SA, Lee JD, Kim BG. Comparison of intratympanic, systemic, and combined steroid therapies for sudden sensorineural hearing loss in patients with diabetes mellitus. Korean J Otorhinolaryngol-Head Neck Surg 2019;62:379-84.

8) Han CS, Park JR, Boo SH, Jo JM, Park KW, Lee WY, et al. Clinical efficacy of initial intratympanic steroid treatment on sudden sensorineural hearing loss with diabetes. Otolaryngol Head Neck Surg 2009; 141:572-8.

9) Weng SF, Chen YS, Liu TC, Hsu CJ, Tseng FY. Prognostic factors of sudden sensorineural hearing loss in diabetic patients. Diabetes Care 2004;27:2560-1.

10) Siegel LG. The treatment of idiopathic sudden sensorineural hearing loss. Otolaryngol Clin North Am 1975;8:467-73.

11) Lee JS, Kim NY, Na SH, Youn YH, Shin CS. Reference values of neutrophil-lymphocyte ratio, lymphocyte-monocyte ratio, plateletlymphocyte ratio, and mean platelet volume in healthy adults in South Korea. Medicine (Baltimore) 2018;97:e11138.
12) Kurien M, Thomas K, Bhanu TS. Hearing threshold in patients with diabetes mellitus. J Laryngol Otol 1989;103:164-8.

13) Wilson WR, Laird N, Moo-Young G, Soeldner JS, Kavesh DA, MacMeel JW. The relationship of idiopathic sudden hearing loss to diabetes mellitus. Laryngoscope 1982;92:155-60.

14) Nemati S, Hassanzadeh R, Mehrdad M, Sajedi Kia S. Hearing status in patients with type 2 diabetes mellitus according to blood-sugar control: a comparative study. Iran J Otorhinolaryngol 2018;30:209-18.

15) Srinivas CV, Shyamala V, Shiva Kumar BR. Clinical study to evaluate the association between sensorineural hearing loss and diabetes mellitus in poorly controlled patients whose $\mathrm{HbAlc}>8$. Indian $\mathrm{J}$ Otolaryngol Head Neck Surg 2016;68:191-5.

16) Maia CA, Campos CA. Diabetes mellitus as etiological factor of hearing loss. Braz J Otorhinolaryngol 2005;71:208-14.

17) Makishima K, Tanaka K. Pathological changes of the inner ear and central auditory pathway in diabetics. Ann Otol Rhinol Laryngol 1971; 80:218-28.

18) Costa OA. Inner ear pathology in experimental diabetes. Laryngoscope 1967;77:68-75.

19) Jun HJ, Hwang SY, Lee SH, Lee JE, Song JJ, Chae S. The prevalence of hearing loss in South Korea: data from a population-based study. Laryngoscope 2015;125:690-4.

20) Weng SF, Chen YS, Hsu CJ, Tseng FY. Clinical features of sudden sensorineural hearing loss in diabetic patients. Laryngoscope 2005; 115:1676-80.

21) Chen L, Zhang G, Zhang Z, Wang Y, Hu L, Wu J. Neutrophil-tolymphocyte ratio predicts diagnosis and prognosis of idiopathic sudden sensorineural hearing loss: a systematic review and meta-analysis. Medicine (Baltimore) 2018;97:e12492.

22) Lou M, Luo P, Tang R, Peng Y, Yu S, Huang W, et al. Relationship between neutrophil-lymphocyte ratio and insulin resistance in newly diagnosed type 2 diabetes mellitus patients. BMC Endocr Disord 2015;15:9.

23) Hussain M, Babar MZM, Akhtar L, Hussain MS. Neutrophil lymphocyte ratio (NLR): a well assessment tool of glycemic control in type 2 diabetic patients. Pak J Med Sci 2017;33:1366-70.

24) Dziedzic T. Systemic inflammation as a therapeutic target in acute ischemic stroke. Expert Rev Neurother 2015;15:523-31.

25) Ermutlu G, Süslü N, Yılmaz T, Saraç S. Sudden hearing loss: an effectivity comparison of intratympanic and systemic steroid treatments. Eur Arch Otorhinolaryngol 2017;274:3585-91.

26) Ahn JH, Kim TY, Kim YJ, Han MW, Yoon TH, Chung JW. Lipoprostaglandin E1 in combination with steroid therapy is effective for treatment of sudden sensorineural hearing loss in Korean patients with type 2 diabetes. Diabet Med 2006;23:1339-43.

27) Ahn JH, Han MW, Kim JH, Chung JW, Yoon TH. Therapeutic effectiveness over time of intratympanic dexamethasone as salvage treatment of sudden deafness. Acta Otolaryngol 2008;128:128-31.

28) Byl FM Jr. Sudden hearing loss: eight years' experience and suggested prognostic table. Laryngoscope 1984;94:647-61.

29) Park MK, Lee CK, Park KH, Lee JD, Lee CG, Lee BD. Simultaneous versus subsequent intratympanic dexamethasone for idiopathic sudden sensorineural hearing loss. Otolaryngol Head Neck Surg 2011; 145:1016-21.

30) Yoo MH, Lim WS, Park JH, Kwon JK, Lee TH, An YH, et al. Simultaneous versus sequential intratympanic steroid treatment for severe-to-profound sudden sensorineural hearing loss. Audiol Neurootol 2016;21:399-405. 